\title{
Genistein attenuates renal fibrosis in streptozotocin-induced diabetic rats
}

\author{
QIANG JIA, RUI YANG, XIAO-FEN LIU, SHAN-FENG MA and LEI WANG \\ Department of Physiology, Bengbu Medical College, Bengbu, Anhui 233030, P.R. China
}

Received January 10, 2018; Accepted October 17, 2018

DOI: $10.3892 / \mathrm{mmr} .2018 .9635$

\begin{abstract}
The present study aimed to investigate the antifibrogenic effects of genistein (GEN) on the kidney in streptozotocin (STZ)-induced diabetic rats and to determine the associated mechanisms. Rats were randomized into four groups: Normal control (N), STZ (S), L (STZ+ low-dose GEN) and $\mathrm{H}(\mathrm{STZ}+$ high-dose GEN). After 8 weeks, the fasting blood glucose (FBG) level, the ratio of kidney weight to body weight (renal index), 24-h urine protein, blood urea nitrogen (BUN), serum creatinine ( $\mathrm{SCr}$ ), renal total antioxidant capacity (T-AOC), superoxide dismutase (SOD), lipid peroxidation (LPO), malondialdehyde (MDA) and hydroxyproline (Hyp) contents were measured. The histomorphology and ultrastructure of the kidney were also assessed. In addition, mRNA expression levels of transforming growth factor- $\beta 1$ (TGF- $\beta 1$ ) and protein expression levels of nuclear factor erythroid 2-related factor 2 (Nrf2), heme oxygenase-1 (HO-1), NAD(P) $\mathrm{H}$ :quinone oxidoreductase 1 (NQO1), TGF- $\beta 1$, mothers against decapentaplegic homolog 3 (Smad3), phosphorylated (p)-Smad3 and collagen IV were estimated. Compared with group $\mathrm{N}$, the levels of FBG, renal index, 24-h urine protein, BUN, SCr, LPO, MDA and Hyp were increased, whereas the levels of T-AOC and SOD were decreased in group S. The structure of renal tissue was damaged, and the expression of Nrf2, HO-1 and NQO1 were reduced, whereas the expression of TGF- $\beta 1$, Smad3, p-Smad3 and collagen IV were increased in group $\mathrm{S}$. Compared with group $\mathrm{S}$, the aforementioned indices were improved in groups $\mathrm{L}$ and $\mathrm{H}$. In conclusion, GEN exhibited reno-protective effects in diabetic rats and its mechanisms may be associated with the inhibition of oxidative stress by activating the Nrf2-HO-1/NQO1 pathway, and the alleviation of renal fibrosis by suppressing the TGF- $\beta 1 / \mathrm{Smad} 3$ pathway.
\end{abstract}

Correspondence to: Ms. Rui Yang, Department of Physiology, Bengbu Medical College, 2600 Dong Hai Avenue, Bengbu, Anhui 233030, P.R. China

E-mail: xzxyr@sina.com

Key words: genistein, diabetes mellitus, rat, renal fibrosis, oxidative stress, transforming growth factor- $\beta 1 /$ mothers against decapentaplegic homolog 3 pathway

\section{Introduction}

Chronic kidney disease is increasing worldwide at an annual rate of $8 \%$, with the prevalence higher in developing countries (1). Diabetic nephropathy (DN) is a common underlying cause (2). $\mathrm{DN}$ is one of the most common microvascular complications of type 1 and type 2 diabetes mellitus (3), and is characterized by persistent proteinuria, progressive loss of renal function and morphological alterations, including glomerular hypertrophy, mesangial expansion, glomerular basement membrane thickening and interstitial fibrosis (4). Although metabolic and hemodynamic alterations, inflammation and activation of the renin-angiotensin system are involved in the pathogenesis of $\mathrm{DN}$, the precise cause remains unclear. Emerging evidence suggests that oxidative stress serves a crucial role in the occurrence and development of DN (5).

The nuclear factor erythroid 2-related factor 2(Nrf2) signaling pathway is a crucial cytoprotective regulator in mammalian cells in response to endogenous and exogenous stress (6). Under physiological conditions, Nrf2 is inactivated by its inhibitory cytosolic protein, Kelch-like ECH-associated protein 1 (Keap1). When cells are exposed to redox modulators, Nrf2 is released from Keap1 and translocates into the nucleus (7). Nrf2, which binds to antioxidant response elements in the nucleus, regulates the transcription of antioxidant genes, including heme oxygenase 1 (HO-1) and NAD(P)H:quinone oxidoreductase 1 (NQO1). The induction of HO-1 and NQO1 has been considered to be adaptive cellular responses to oxidative stress (8).

Oxidative stress is defined as a disturbance in the balance between the production of reactive oxygen species (ROS) and antioxidant defenses (9). Diabetes may increase ROS production through a variety of pathways, including strengthening the autoxidation of glucose and increasing the formation of superoxides and advanced glycation end products (10). Excess amounts of ROS induce the activation of various transcription factors that increase the synthesis of extracellular matrix (ECM) and renal fibrosis, eventually leading to end-stage renal disease (11). A previous study demonstrated that renal fibrosis is one of the main pathological alterations in DN (12). Another report suggested that excess ROS may upregulate transforming growth factor- $\beta$ (TGF- $\beta$ ) expression, which leads to over-production of ECM, thickening of the glomerular basement membrane and renal fibrosis (13). Therefore, the TGF- $\beta$ family is considered an important pro-fibrotic mediator in fibrotic diseases (14). 
Genistein (GEN) is a major isoflavone in soybeans that interacts with estrogen receptors in vivo and is a phytoestrogen (15). GEN has attracted considerable attention in previous years owing to its reported beneficial health effects, such as the prevention of a number of chronic diseases, including osteoporosis, cardiovascular diseases, diabetes and cancer (16-19). Previous studies have demonstrated that GEN has anti-inflammatory, anti-oxidative, anti-apoptotic and anti-proliferative effects (20-22). Our previous study demonstrated that GEN attenuated myocardial fibrosis in rats with streptozotocin (STZ)-induced type 1 DM (T1DM) by inhibiting the overexpression of collagen I and collagen III, and by suppressing the TGF- $\beta /$ mothers against decapentaplegic homolog 3 (Smad3) pathway (23). A recent study demonstrated that GEN reduces renal ischemia/reperfusion-induced cell death by inhibiting apoptosis (24). In addition, in high fructose-fed rats, GEN significantly inhibited the production of inflammatory cytokines and reduced the deposition of collagen in renal tissue (13).

Although the positive effects of GEN on renal function are well known, the effects of GEN on renal fibrosis in T1DM remain unclear. Therefore the present study aimed to investigate whether GEN exerts an antifibrogenic effect on T1DM kidney and to determine its associated mechanism.

\section{Materials and methods}

Animals. A total of 24 male Sprague-Dawley rats (age, 6-7 weeks; weight, 160-200 g) were obtained from the Animal Administration Center of Bengbu Medical College (Bengbu, China). The rats were housed in conventional animal facility with a 12-h light/dark cycle at a constant temperature of $21-23^{\circ} \mathrm{C}$ and $50-60 \%$ relative humidity. All the rats were fed with normal laboratory rodent diet and water ad libitum. The present study was approved by the Animal Ethics Committee of Bengbu Medical College.

Study design. Rats were randomized into four groups ( $\mathrm{n}=6$ rats/group): i) Normal control group (N); ii) STZ group (S); iii) STZ+ low-dose GEN group (L); and iv) STZ + high-dose GEN group (H). T1DM was induced as previously described (18). Briefly, rats were deprived of food but had free access to water for $12 \mathrm{~h}$, and subsequently received a single intraperitoneal (i.p.) injection of STZ $(55 \mathrm{mg} / \mathrm{kg}$ body weight; Sigma-Aldrich, Merck KGaA, Darmstadt, Germany) freshly dissolved in $0.1 \mathrm{~mol} / 1$ citrate buffer. Rats in the $\mathrm{N}$ group received an i.p. injection of a similar volume of citrate buffer. Rats with fasting blood glucose (FBG) level $>16.7 \mathrm{mmol} / \mathrm{l}$ (3 days post-injection) indicated successful establishment of the T1DM model. From the fifth week following model establishment, rats in groups $\mathrm{L}$ and $\mathrm{H}$ received a daily gavage with 5 and $25 \mathrm{mg} / \mathrm{kg}$ GEN (Sigma-Aldrich; Merck KGaA) freshly dissolved in carboxymethylcellulose sodium (CMC-Na) solution (Sangon Biotech, Co., Ltd., Shanghai, China), respectively, up to the end of the week 8 . Rats in groups $\mathrm{N}$ and $\mathrm{S}$ were received a daily gavage with a similar volume of CMC-Na solution over the same time period.

Determination of $F B G, 24-h$ urine protein, blood urea nitrogen (BUN) and serum creatinine (SCr) levels. At 8 weeks post-induction, the rats were placed in metabolic cages for 24-h urine collection and consequent albuminuria measurement prior to being anesthetized with chloral hydrate $(400 \mathrm{mg} / \mathrm{kg}$; i.p.). Blood was obtained from the tail vein to measure FBG using a portable glucometer (Accu-Chek, Roche, Mannheim, Germany). Then, $4 \mathrm{ml}$ blood from abdominal aorta was collected. The fresh blood was placed in serum tubes and centrifuged at $765 \mathrm{x} \mathrm{g}$ for $20 \mathrm{~min}$ at $4^{\circ} \mathrm{C}$. The serum was collected and the levels of 24-h urine protein, BUN and $\mathrm{SCr}$ were measured using a Urine Protein Test Kit (cat. no. C035-2), Urea Assay Kit (cat. no. C013-2) and Creatinine Assay Kit (cat. no. C011-2; Jiancheng Bioengineering Institute, Nanjing, Jiangsu, China) according to the manufacturer's protocols.

Detection of body weight $(B W)$, kidney weight $(K W)$ and renal index. Rats were sacrificed and their bilateral kidneys were excised and placed in ice-cold normal saline, and the ratio of kidney weight to body weight (renal index) was calculated.

Determinationoftotalantioxidantcapacity (T-AOC), superoxide dismutase (SOD), lipid peroxidation (LPO), malondialdehyde (MDA) and hydroxyproline (Hyp) levels. Renal tissue (0.1 g) was homogenized in $0.9 \mathrm{ml}$ normal saline. The supernatant fluid was collected following centrifugation for $20 \mathrm{~min}(825 \mathrm{x} \mathrm{g}$ at $4^{\circ} \mathrm{C}$ ). Following measurement of the protein concentration using a Bicinchoninic Acid (BCA) Protein Assay kit (cat. no. P0010; Beyotime Institute of Biotechnology, Shanghai, China), the renal T-AOC, SOD, LPO, MDA and Hyp levels were measured according to the protocols of Total Antioxidant Capacity Assay kit (cat. no. A015-2), Total Superoxide Dismutase Assay kit (cat. no. A001-1-1), Lipid Peroxidation Assay kit (cat. no. A106), Malondialdehyde Assay kit (cat. no. A003-1) and Hydroxyproline Assay kit (cat. no. A030-3; Nanjing Jiancheng Bioengineering Institute, Nanjing, China).

Histological examination. For histological analysis, fresh left renal tissue collected from each group was fixed using $4 \%$ paraformaldehyde for $12 \mathrm{~h}$ at $4^{\circ} \mathrm{C}$. Tissues were embedded in paraffin, cut into 5- $\mu \mathrm{m}$ thick section and stained using hematoxylin for $5 \mathrm{~min}$ and $0.5 \%$ eosin for $2 \mathrm{~min}$ (H\&E) at room temperature. For Masson's trichrome staining, paraffin sections were dewaxed with xylene, rehydrated with graded ethanol at room temperature and stained with Regaud dye hematoxylin for $10 \mathrm{~min}$ at room temperature. Following rinsing with water, sections were stained with Ponceau Fuchsin acid solution for $10 \mathrm{~min}$ at room temperature, immersed in $2 \%$ acetic acid aqueous solution and treated with a $1 \%$ aqueous solution of phosphomolybdic acid for $5 \mathrm{~min}$ at room temperature. Without rinsing with water, the sections were stained with $2 \%$ aniline blue for $5 \mathrm{~min}$ at room temperature, immersed in $0.2 \%$ acetic acid aqueous solution, in $95 \%$ alcohol, anhydrous alcohol, permeabilized with xylene and mounted with neutral resin. The sections were observed and images were captured using a NanoZoomer 2.0 RS Digital Pathology slide scanner (Hamamatsu Photonics K.K., Hamamatsu, Japan). Renal collagen volume fraction (CVF) was analyzed using Image-Pro Plus 6.0 analysis software (Media Cybernetics, Inc., Rockville, MD, USA). A total of five fields of view per sample were randomly chosen and the average was determined for analysis. 
Ultrastructure observation. Fresh left kidneys were cut into $1 \times 1 \times 1 \mathrm{~mm}$ cubes and fixed using $2.5 \%$ glutaraldehyde for 4-6 $\mathrm{h}$ at $4^{\circ} \mathrm{C}$. Tissues were washed with $0.1 \mathrm{~mol} / 1$ phosphate buffer and post-fixed in $1 \%$ osmium tetroxide for $1 \mathrm{~h}$ at room temperature. Tissues were embedded in Epon812 for $2 \mathrm{~h}$ at room temperature, put into an oven at $45^{\circ} \mathrm{C}$ for $12 \mathrm{~h}$ and at $65^{\circ} \mathrm{C}$ for $48 \mathrm{~h}$ then cut into 70 -nm thick ultrathin sections and stained with uranyl acetate for $30 \mathrm{~min}$ and lead citrate for $15 \mathrm{~min}$ at room temperature. These sections were examined using a JEM-1230 JEOL transmission electron microscope (TEM; Tokyo, Japan).

Semi-quantitative reverse transcription-polymerase chain reaction ( $R T-P C R)$. The mRNA expression levels of TGF- $\beta 1$ in renal tissue were determined by RT-PCR. Briefly, total RNA was extracted from renal tissue $(0.1 \mathrm{~g})$ using TRIzol ${ }^{\circledR}$ (Invitrogen; Thermo Fisher Scientific, Inc., Waltham, MA, USA), according to the manufacturer's protocol. cDNA was synthesized according to the protocol of RevertAid First Strand cDNA Synthesis kit (cat. no. K1622; Invitrogen; Thermo Fisher Scientific, Inc.). TGF- $\beta 1$ and $\beta$-actin genes were amplified using PCR Master Mix (cat. no. K0171; Thermo Fisher Scientific, Inc.). All PCR reactions were performed with a T-Gradient thermocycler (Biometra GmbH, Göttingen, Germany). The primer sequences were as follows: TGF- $\beta 1$, forward 5'-CCAAGGAGACGGAATACAGG-3', reverse 5'-ATGAGGAGCAGGAAGGGTC-3' (expected size, 156 bp); $\beta$-actin, forward 5'-GATGGTGGGTATGGGTCAGAAGGA GG-3', reverse 5'-GCTCATTGCCGATAGTGATGACC-3' (expected size, $632 \mathrm{bp}$ ). The thermocycling conditions were as follows: Initial denaturation at $95^{\circ} \mathrm{C}$ for $3 \mathrm{~min}$; followed by 32 cycles of denaturation at $95^{\circ} \mathrm{C}$ for $50 \mathrm{sec}$; annealing at $54.5^{\circ} \mathrm{C}(\mathrm{TGF}-\beta 1)$ or $59.4^{\circ} \mathrm{C}(\beta$-actin) for $50 \mathrm{sec}$; and extension at $72^{\circ} \mathrm{C}$ for $60 \mathrm{sec}$; followed by final extension at $72^{\circ} \mathrm{C}$ for $10 \mathrm{~min}$. The PCR products were electrophoresed in $1.5 \%$ agarose gel and stained with ethidium bromide. Densitometric analysis of TGF- $\beta 1$ gene expression was normalized to the corresponding $\beta$-actin gene using Tanon 3.1.2 software (Tanon Science and Technology, Co., Ltd., Shanghai, China).

Western blotting. Rat renal tissue $(0.1 \mathrm{~g})$ was lysed using Cell Lysis Buffer for western blotting and IP (cat. no. P0013; Beyotime Institute of Biotechnology) with phenylmethanesulfonyl fluoride (cat. no. ST506; Beyotime Institute of Biotechnology). The protein concentration was measured using the BCA Protein Assay kit. A total of $50 \mu \mathrm{g}$ protein was separated by $10 \%$ SDS-PAGE and subsequently electroblotted onto polyvinylidene difluoride membranes. Membranes used for $\mathrm{Nrf} 2$, HO- 1 , NQO1, TGF- $\beta 1$, Smad3, collagen-IV and GAPDH detection were blocked in TBS $+0.1 \%$ Tween 20 (TBST) containing $5 \%$ nonfat dry milk at $37^{\circ} \mathrm{C}$ for $2 \mathrm{~h}$, and membranes used for phosphorylated (p)-Smad3 were blocked in TBST containing 5\% bovine serum albumin (cat. no. B2064; Sigma-Aldrich; Merck $\mathrm{KGaA}$ ) at $37^{\circ} \mathrm{C}$ for $2 \mathrm{~h}$. The membranes were incubated with primary antibodies against the following proteins: Nrf2 (1:1,000; cat. no. ab137550; Abcam, Cambridge, MA, USA), HO-1 (1:1,000; cat. no. ab52947; Abcam), NQO1 (1:300; cat. no. PB0526; Wuhan Boster Biological Technology, Ltd., Wuhan, China), TGF- $\beta 1$ (1:1,000; cat. no. ab92486; Abcam), Smad3 (1:1,000; cat. no. ab40854; Abcam), p-Smad3 (1:1,000;
Table I. Alterations of BW, KW and renal index in diabetic model rats.

\begin{tabular}{lllc}
\hline Group & BW $(\mathrm{g})$ & KW $(\mathrm{mg})$ & Renal index $^{\mathrm{a}}(\mathrm{mg} / \mathrm{g})$ \\
\hline $\mathrm{N}$ & $421.54 \pm 33.62$ & $2.92 \pm 0.22$ & $7.02 \pm 0.56$ \\
$\mathrm{~S}$ & $232.15 \pm 23.07^{\mathrm{b}}$ & $3.52 \pm 0.15^{\mathrm{b}}$ & $13.70 \pm 0.64^{\mathrm{b}}$ \\
L & $257.46 \pm 27.94$ & $3.42 \pm 0.12$ & $12.78 \pm 0.62^{\mathrm{c}}$ \\
H & $290.30 \pm 31.28^{\mathrm{d}}$ & $3.28 \pm 0.14^{\mathrm{c}}$ & $11.88 \pm 0.46^{\mathrm{d}}$ \\
\hline
\end{tabular}

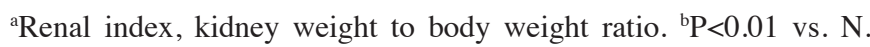
${ }^{\mathrm{c}} \mathrm{P}<0.05$ vs. $\mathrm{S} .{ }^{\mathrm{d}} \mathrm{P}<0.01$ vs. $\mathrm{S}$. Data are presented as the mean \pm standard deviation; $\mathrm{n}=6$. BW, body weight; $\mathrm{H}$, streptozotocin + high-dose genistein group; KW, kidney weight; L, streptozotocin +low-dose genistein group; N, normal control group; S, streptozotocin group.

cat. no. ab52903; Abcam), collagen IV (1:300; cat. no. A01411; Wuhan Boster Biological Technology, Ltd.) and GAPDH (1:2,000; cat. no. ab181602; Abcam) at $4^{\circ} \mathrm{C}$ overnight, followed by incubation with horseradish peroxidase-conjugated goat anti-rabbit immunoglobulin G secondary antibody (1:2,000; cat. no. BL003A; Biosharp Biotechnology, Hefei, China) for $1 \mathrm{~h}$ at room temperature. The blots were detected using an enhanced chemiluminescent reagent (cat. no. WBKLS0100; Millipore Corporation, Billerica, MA, USA) and scanned with ChemiDoc XRS system (Bio-Rad Laboratories, Inc., Hercules, CA, USA). The intensities of the protein bands were quantified and normalized to GAPDH using Quantity One Software Version 4.6.6 (Bio-Rad Laboratories, Inc.).

Statistical analysis. All statistical analysis was conducted using SPSS Software, version 17.0 (SPSS, Inc., Chicago, IL, USA). All tests were repeated three times. Data are expressed as the mean \pm standard deviation. Statistical comparisons were analyzed using one-way analysis of variance followed by the Newman-Keuls test. $\mathrm{P}<0.05$ was considered to indicate a statistically significant difference.

\section{Results}

Alterations of $F B G, 24-h$ urine protein, $B U N$ and $\mathrm{SCr}$ levels. The levels of FBG, 24-h urine protein, BUN and $\mathrm{SCr}$ were significantly increased in the $\mathrm{S}$ group compared with the respective levels in the $\mathrm{N}$ group $(\mathrm{P}<0.01$; Fig. 1A-D). No significant differences were identified in FBG levels between rats in the $\mathrm{L}$ or $\mathrm{H}$ group compared with those in the $\mathrm{S}$ group (Fig. 1A); conversely, BUN, 24-h urine protein and $\mathrm{SCr}$ levels were significantly decreased in the $\mathrm{L}$ and $\mathrm{H}$ groups compared with the $\mathrm{S}$ group $(\mathrm{P}<0.05, \mathrm{P}<0.01$; Fig. 1B-D).

Effects on $B W, K W$ and renal index. Compared with group N, BW was significantly decreased $(\mathrm{P}<0.01$; Table I), whereas $\mathrm{KW}$ and renal index were significantly increased in the $\mathrm{S}$ group $(\mathrm{P}<0.01)$. Compared with group $\mathrm{S}$, there were no significant differences in $\mathrm{BW}$ and $\mathrm{KW}$ in group L, whereas renal index was significantly decreased $(\mathrm{P}<0.05)$. BW was significantly increased $(\mathrm{P}<0.01)$, $\mathrm{KW}$ and renal index were significantly decreased $(\mathrm{P}<0.05$ and $\mathrm{P}<0.01$, respectively) in group $\mathrm{H}$ compared with group $\mathrm{S}$ (Table I). 
Table II. Levels of T-AOC, SOD, LPO, MDA and Hyp in renal tissues of diabetic model rats.

\begin{tabular}{lccccl}
\hline Group & T-AOC $(\mathrm{U} / \mathrm{mg})$ & SOD $(\mathrm{U} / \mathrm{mg})$ & LPO $(\mu \mathrm{mol} / \mathrm{g})$ & MDA $(\mathrm{nmol} / \mathrm{mg})$ & Hyp $(\mu \mathrm{g} / \mathrm{mg})$ \\
\hline $\mathrm{N}$ & $87.55 \pm 6.97$ & $64.10 \pm 7.95$ & $15.24 \pm 2.62$ & $3.78 \pm 0.49$ & $2.95 \pm 0.28$ \\
$\mathrm{D}$ & $51.65 \pm 5.52^{\mathrm{a}}$ & $31.22 \pm 4.70^{\mathrm{a}}$ & $31.46 \pm 3.41^{\mathrm{a}}$ & $6.92 \pm 0.72^{\mathrm{a}}$ & $5.67 \pm 0.55^{\mathrm{a}}$ \\
$\mathrm{L}$ & $60.12 \pm 5.68^{\mathrm{b}}$ & $39.56 \pm 5.24^{\mathrm{b}}$ & $27.19 \pm 3.23^{\mathrm{b}}$ & $6.18 \pm 0.54^{\mathrm{b}}$ & $5.13 \pm 0.43^{\mathrm{b}}$ \\
$\mathrm{H}$ & $67.49 \pm 6.11^{\mathrm{c}}$ & $47.83 \pm 5.58^{\mathrm{c}}$ & $22.65 \pm 2.87^{\mathrm{c}}$ & $5.72 \pm 0.53^{\mathrm{c}}$ & $4.46 \pm 0.41^{\mathrm{c}}$ \\
\hline
\end{tabular}

${ }^{\mathrm{a}} \mathrm{P}<0.01$ vs. N. ${ }^{\mathrm{b}}<<0.05$ vs. $\mathrm{S} .{ }^{\mathrm{c}} \mathrm{P}<0.01$ vs. $\mathrm{S}$. Data are presented as the mean \pm standard deviation; $\mathrm{n}=6$. H, streptozotocin + high-dose genistein group; Hyp, hydroxyproline; L, streptozotocin + low-dose genistein group; LPO, lipid peroxidation; MDA, malondialdehyde; N, normal control group; S, streptozotocin group; SOD, superoxide dismutase; T-AOC, total antioxidant capacity.
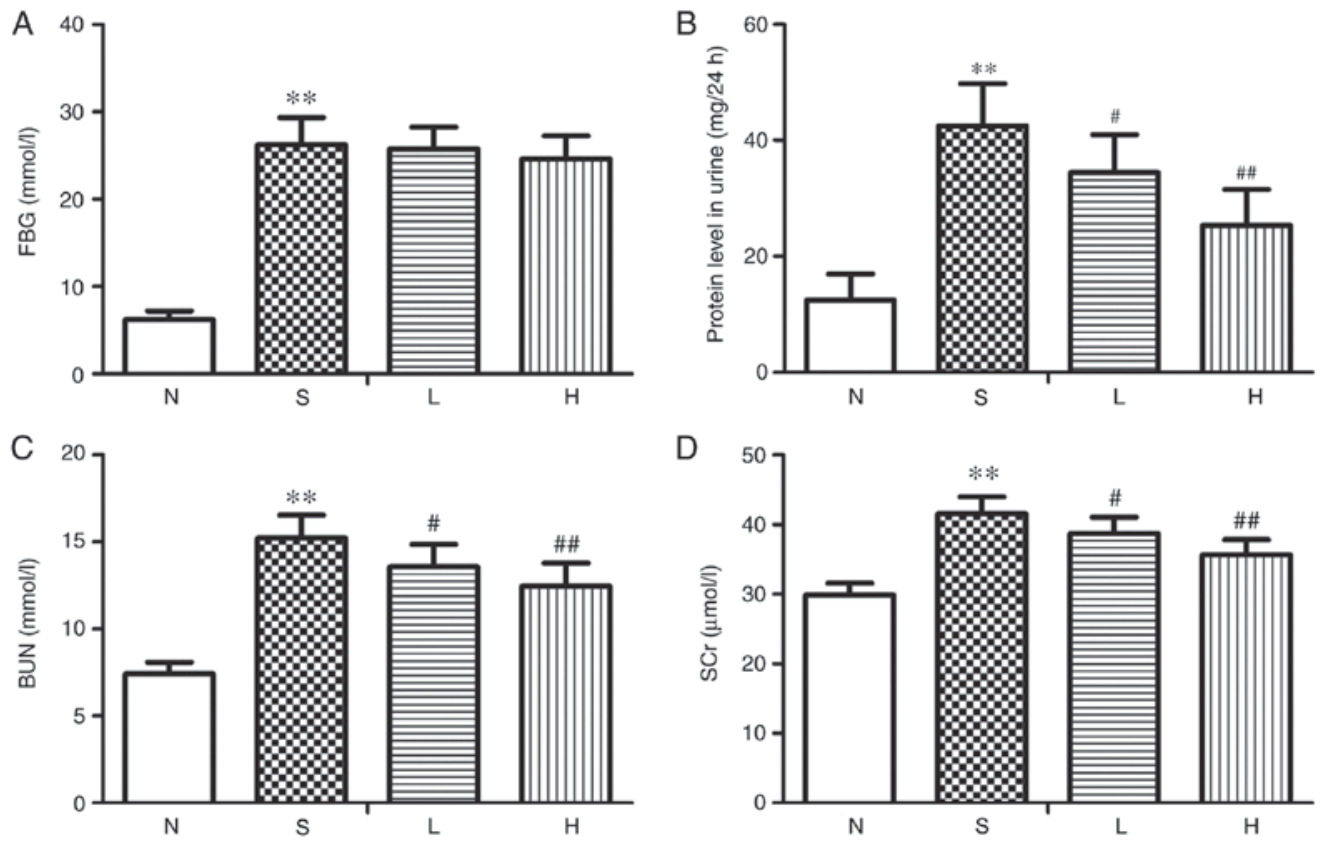

Figure 1. Levels of kidney function markers in rats. (A) FBG, (B) 24-h urine protein, (C) BUN and (D) SCr level in rats. Data are presented as the mean \pm standard deviation; $\mathrm{n}=6$; ${ }^{* *} \mathrm{P}<0.01$ vs. $\mathrm{N} ;{ }^{~} \mathrm{P}<0.05$ and ${ }^{\# \# /} \mathrm{P}<0.01$ vs. S. BUN, blood urea nitrogen; FBG, fasting blood glucose; H, streptozotocin + high-dose genistein group; L, streptozotocin + low-dose genistein group; N, normal control group; S, streptozotocin group; SCr, serum creatinine.

Effects on T-AOC, SOD, LPO, MDA and Hyp content. The levels of T-AOC and SOD in renal tissue were significantly decreased $(\mathrm{P}<0.01$; Table II), whereas the LPO, MDA and Hyp content were significantly increased $(\mathrm{P}<0.01)$ in group $\mathrm{S}$, compared with the $\mathrm{N}$ control group. Rats in the $\mathrm{L}$ and $\mathrm{H}$ groups exhibited significantly increased levels of T-AOC and SOD $(\mathrm{P}<0.05)$ and significantly decreased LPO, MDA and Hyp content compared with group $\mathrm{S}(\mathrm{P}<0.05$ and $\mathrm{P}<0.01$, respectively; Table II).

Histological alterations in renal tissue. H\&E staining indicated that the glomerular structure was normal and the thickness of glomerular basement membrane was uniform in the kidneys of rats in group $\mathrm{N}$ (Fig. 2). Conversely, the glomerular basement membrane was thickened and the mesangial matrix was increased in group S. Similarly, glomerular basement membrane thickening and mesangial matrix expansion were observed in group L. In group $\mathrm{H}$, the glomerular morphology was improved and pathological alterations were notably decreased compared with group S (Fig. 2).
Morphology of collagen deposition in renal tissue. Masson's trichrome staining demonstrated normal glomerular and renal tubules with less collagen deposition in group $\mathrm{N}$ kidneys (Fig. 3A). A number of collagen deposits in the glomerular perivasular region and intraglomerular region were observed in group S. Compared with group $\mathrm{S}$, collagen deposition was decreased in groups L and H (Fig. 3A). Quantitative analysis indicated that the content of CVF was significantly increased in group $\mathrm{S}$ compared with group $\mathrm{N}(\mathrm{P}<0.01$; Fig. 3B). However, compared with group S, CVF content was significantly decreased in groups $\mathrm{L}$ and $\mathrm{H}(\mathrm{P}<0.05$ and $\mathrm{P}<0.01$, respectively; Fig. 3B).

Alterations in renal ultrastructure. In group N kidneys, TEM demonstrated that the normal glomerular basement membrane and mesangial matrix, as well the podocyte foot processes were neatly arranged (Fig. 4). In the S group, the basement membrane of the cortex was thickened, the mesangial matrix was increased and a number of foot processes were fused. The glomerular basement membrane was thickened and a few foot 

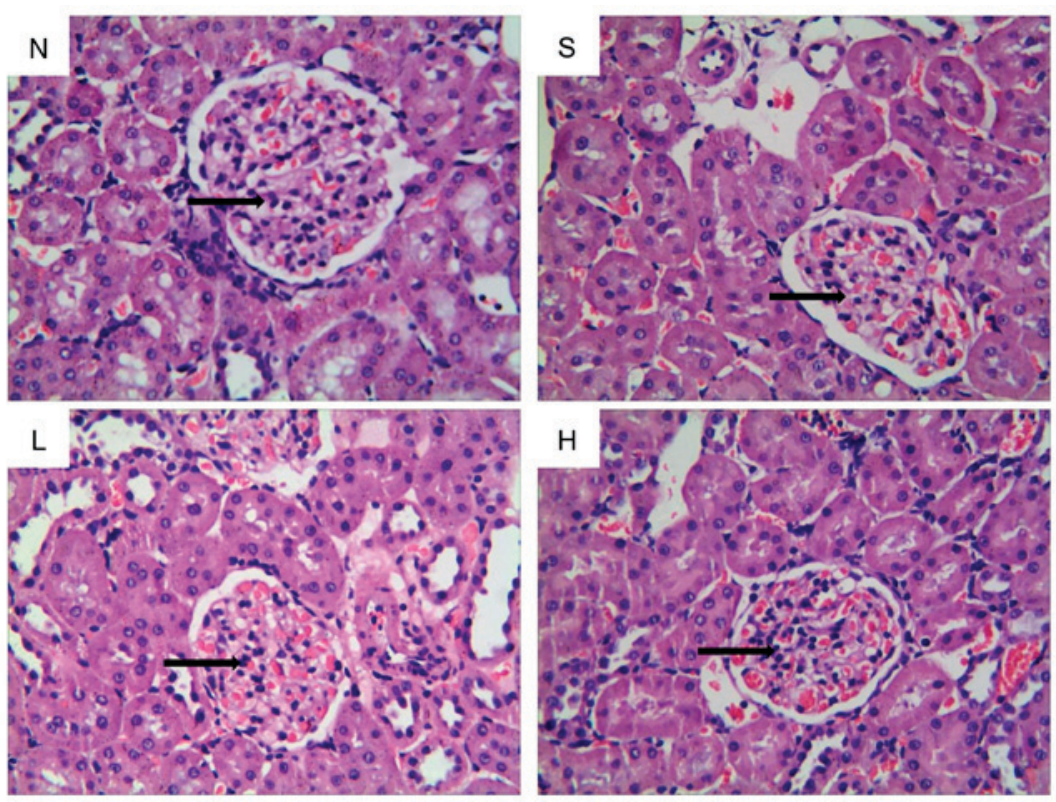

Figure 2. GEN improved the pathological state of renal tissue from diabetic rats. Morphology of renal tissues by hematoxylin and eosin staining; magnification, $\mathrm{x} 400 . \mathrm{N}$ group: The glomerular structure was normal. $\mathrm{S}$ group: The glomerular structure was damaged. $\mathrm{L}$ group: Damage to the glomerular structure was ameliorated. H group: Damage to the glomerular structure was notably ameliorated. Arrows indicate the glomerular structure. $\mathrm{H}$, streptozotocin + high-dose genistein group; L, streptozotocin + low-dose genistein group; N, normal control group; S, streptozotocin group.

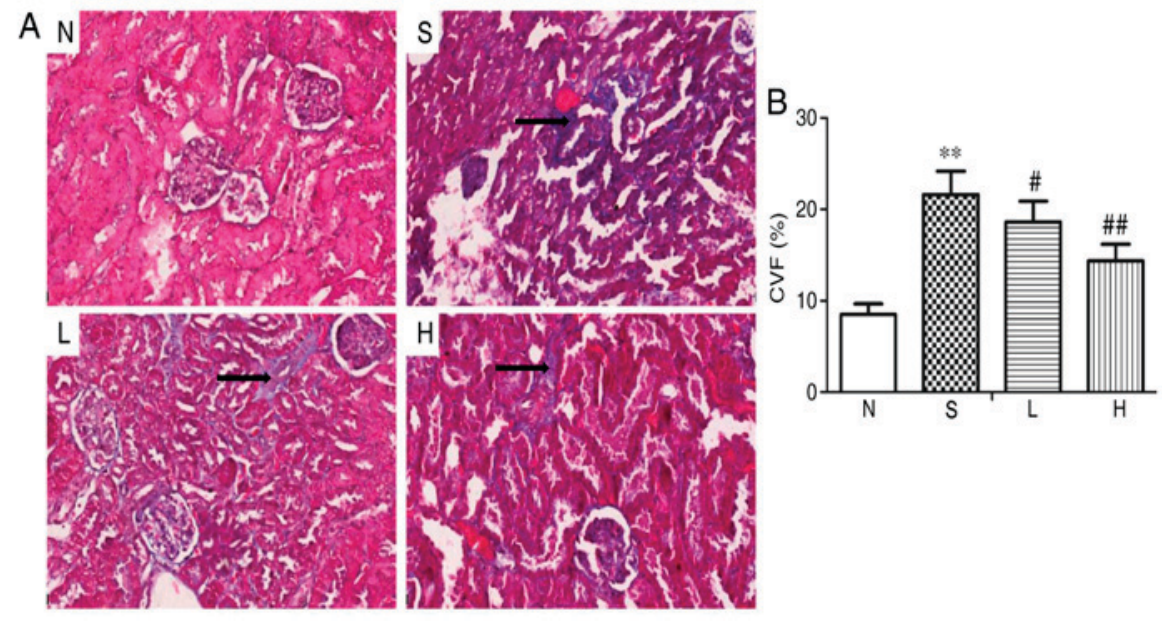

Figure 3. Effects of GEN on renal fibrosis. (A) Representative Masson's trichrome stained tissues from each group; magnification, x200. Arrows indicate collagen. (B) Semi-quantitative analysis of CVF, based on (A). Data are presented as the mean \pm standard deviation; $n=6 ;{ }^{* *} \mathrm{P}<0.01$ vs. N; $\mathrm{P}<0.05$ and ${ }^{\# \# "} \mathrm{P}<0.01$ vs. S. CVF, collagen volume fraction; GEN, genistein; H, streptozotocin + high-dose GEN group; L, streptozotocin + low-dose GEN group; N, normal control group; S, streptozotocin group.
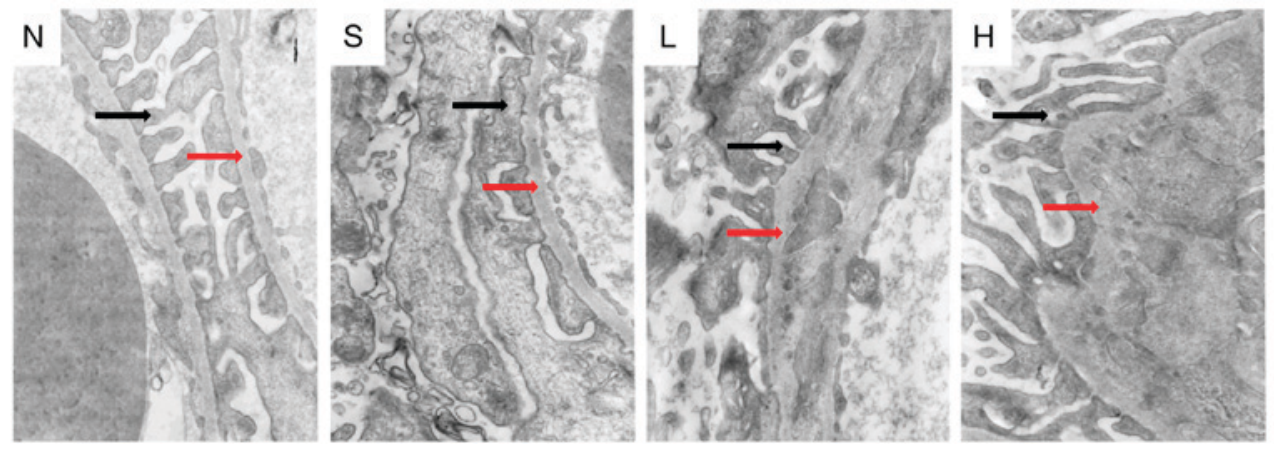

Figure 4. GEN improved the ultrastructure of renal tissue from diabetic rats. Alterations of renal ultrastructure under transmission electron microscopy in rats; magnification, $\mathrm{x} 15,000$. Red arrows indicate the basement membrane and black arrows indicate the podocyte foot processes. $\mathrm{N}$ group: The normal glomerular basement membrane and the podocyte foot processes were neatly arranged. S group: The basement membrane was thickened and foot processes were fused. L group: The fusion of foot processes were ameliorated. $\mathrm{H}$ group: The fusion of foot processes were ameliorated notably. H, streptozotocin + high-dose genistein group; L, streptozotocin + low-dose genistein group; N, control group; S, streptozotocin group. 

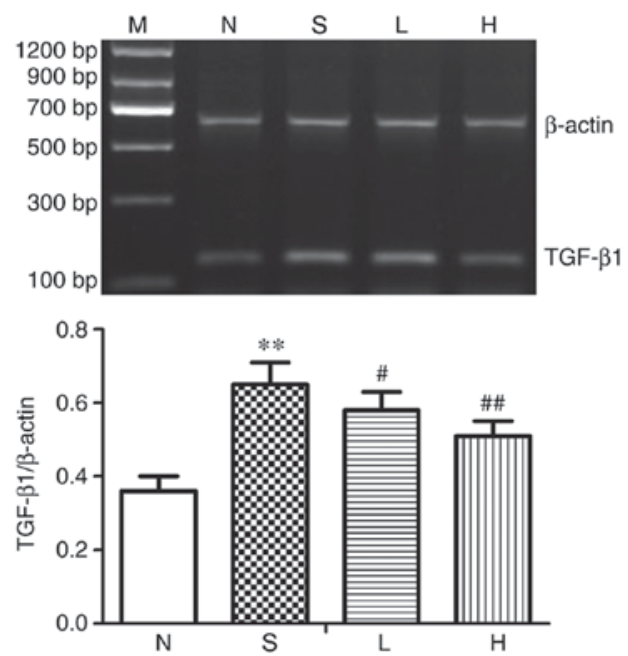

Figure 5. Expression levels of renal TGF- $\beta 1$ mRNA in the different groups. Representative images and semi-quantitative analysis of TGF- $\beta 1$ mRNA expression levels, as determined by reverse transcription-polymerase chain reaction, in renal tissues of rats. Data are presented as the mean \pm standard deviation; $\mathrm{n}=6 ;{ }^{* *} \mathrm{P}<0.01$ vs. $\mathrm{N} ;{ }^{\#} \mathrm{P}<0.05$ and ${ }^{\# \#} \mathrm{P}<0.01$ vs. $\mathrm{S}$. $\mathrm{H}$, streptozotocin + high-dose genistein group; L, streptozotocin + low-dose genistein group; $\mathrm{N}$, normal control group; $\mathrm{S}$, streptozotocin group; TGF- $\beta 1$, transforming growth factor- $\beta 1$.
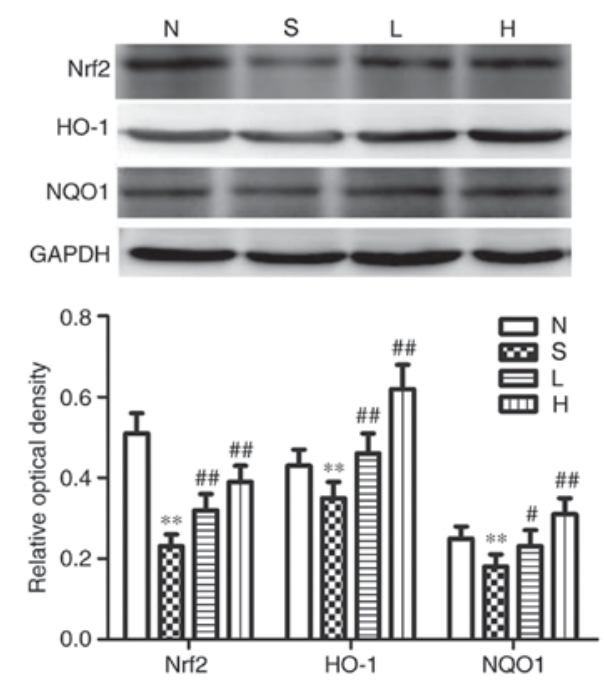

Figure 6. Protein expression levels of Nrf2, HO-1 and NQO1 in the different groups. Representative western blotting images and semi-quantitative analysis of Nrf2, HO-1 and NQO1 proteins; GAPDH was used as a loading control. Data are presented as the mean \pm standard deviation; $n=6 ;{ }^{* *} \mathrm{P}<0.01$ vs. $\mathrm{N}$; ${ }^{\# P} \mathrm{P}<0.05$ and ${ }^{\# \#} \mathrm{P}<0.01$ vs. $\mathrm{S}$. H, STZ+high-dose GEN group; $\mathrm{L}$, STZ+ low-dose GEN group; HO-1, heme oxygenase-1; N, normal control group; Nrf2, nuclear factor erythroid 2-related factor 2; NQO1, NAD(P) $\mathrm{H}$ :quinone oxidoreductase $1 ; \mathrm{S}, \mathrm{STZ}$ group.

processes were fused in group L; whereas, in group $\mathrm{H}$, the glomerular basement membrane and fusion of foot processes were notably improved compared with group S (Fig. 4).

Alterations in renal TGF- $\beta 1$ at the mRNA level. The mRNA expression levels of TGF- $\beta 1$ in renal tissue were significantly upregulated in group $\mathrm{S}$ compared with group $\mathrm{N}(\mathrm{P}<0.01$; Fig. 5). Compared with group S, TGF- $\beta 1$ mRNA expression was significantly decreased in groups $\mathrm{L}$ and $\mathrm{H}(\mathrm{P}<0.05$ and $\mathrm{P}<0.01$, respectively; Fig. 5).
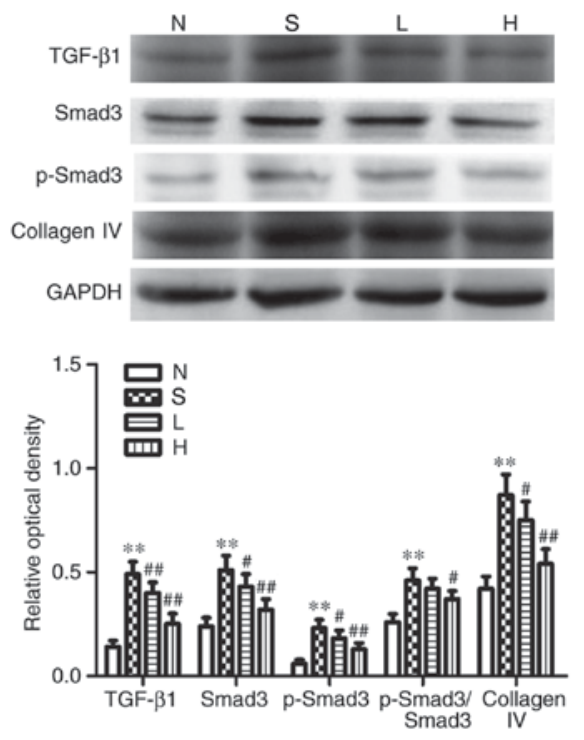

Figure 7. Protein expression of TGF- $\beta 1$, Smad $3, \mathrm{p}-\mathrm{Smad} 3$ and collagen IV in the different groups. Representative western blotting images and semi-quantitative analysis of TGF- $\beta 1, \mathrm{Smad} 3, \mathrm{p}-\mathrm{Smad} 3, \mathrm{p}-\mathrm{Smad} 3 / \mathrm{Smad} 3$ and collagen IV proteins; GAPDH was used as a loading control. Data are presented as the mean \pm standard deviation; $n=6 ;{ }^{* *} \mathrm{P}<0.01$ vs. $\mathrm{N} ;{ }^{*} \mathrm{P}<0.05$ and ${ }^{\# \#} \mathrm{P}<0.01$ vs. S. H, streptozotocin + high-dose genistein group; L, streptozotocin + low-dose genistein group; N, normal control group; $\mathrm{p}$, phosphorylated; S, streptozotocin group; Smad3, mothers against decapentaplegic homolog 3 ; TGF- $\beta 1$, transforming growth factor- $\beta 1$.

Effects on renal Nrf2, HO-1, NQO1, TGF- $\beta 1$, Smad3, p-Smad3 and collagen-IV at protein expression levels. The protein expression levels of renal Nrf2, HO-1 and NQO1 were significantly reduced compared with the respective expression levels in the $\mathrm{N}$ group $(\mathrm{P}<0.01$; Fig. 6$)$, whereas the protein expression levels of TGF- $\beta 1$, Smad3, p-Smad3 and collagen IV, as well as the $\mathrm{p}-\mathrm{Smad} 3 / \mathrm{Smad} 3$ ratio were significantly elevated in group $\mathrm{S}$, compared with the $\mathrm{N}$ group $(\mathrm{P}<0.01$; Fig. 7). Compared with group $\mathrm{S}$, the protein expression levels of Nrf2, HO-1 and NQO1 were significantly elevated $(\mathrm{P}<0.05$; Fig. 6), and the protein expression levels of TGF- $\beta 1, \mathrm{Smad} 3, \mathrm{p}$-Smad3 and collagen IV were significantly reduced $(\mathrm{P}<0.05$; Fig. 7$)$ in group L; no significant difference in $\mathrm{p}-\mathrm{Smad} 3 / \mathrm{Smad} 3$ ratio was identified between the $\mathrm{L}$ and $\mathrm{S}$ groups. In group $\mathrm{H}$, the protein expression levels of Nrf2, HO-1, and NQO1 were significantly elevated $(\mathrm{P}<0.01$; Fig. 6$)$, and the protein expression levels of TGF- $\beta 1$, Smad3, p-Smad 3 and collagen-IV, as well as the p-Smad3/Smad3 ratio were significantly reduced compared with $\mathrm{S}(\mathrm{P}<0.05$; Figs. 6 and 7$)$.

\section{Discussion}

DN is a major diabetic microvascular complication and is the leading cause of chronic renal failure and end-stage renal disease (25). The main pathological alterations in DN are increased mesangial matrix in the glomerulus, basement membrane thickening and interstitial fibrosis (26). In the present study, rats received a large i.p. dose of STZ to induce a T1DM model. The results demonstrated that BW was reduced, and the $\mathrm{FBG}, \mathrm{KW}$, renal index, 24-h urine protein, BUN and $\mathrm{SCr}$ levels were increased in group S. In addition, $\mathrm{H} \& \mathrm{E}$ staining and ultrastructure observations demonstrated that 
glomerular basement membrane thickening and the mesangial matrix increased in the diabetic renal tissue, which indicated that the DN model was successfully established, and that renal hypertrophy and dysfunction were aggravated in the diabetic model rats.

Oxidative stress serves a pivotal role in the occurrence and development of DN (27). The imbalance between oxidation and anti-oxidant systems, and excess ROS generation are the main pathogenic factors in renal disease (28). It has been reported that hyperglycemia may induce the glomerular mesangial cells and tubular epithelial cells to produce excess ROS, which destroy tissue proteins, produces a large quantity of lipid peroxides and further aggravates renal oxidative damage (29). T-AOC reflects the total antioxidant capacity of the body's defense system, and LPO and MDA reflect the levels of tissue lipid peroxidation. SOD functions to scavenge superoxide products, which reflects the intracellular reductive capacity. In the present study, the results demonstrated that compared with normal control rats, diabetic rats exhibited reduced T-AOC and SOD activities and their LPO and MDA contents were increased. These results indicated that diabetes may decrease renal total antioxidant capacity and increase the production of lipid peroxides, which may lead to renal oxidative stress injury. In addition, western blotting results demonstrated that the protein expression of Nrf2, HO-1 and NQO1 were reduced, which suggested that the Nrf2-HO-1/NQO1 pathway may be involved in diabetes-induced oxidative stress.

GEN is a plant phytoestrogen that has an anti-oxidative effect. For example, a previous study reported that GEN prevents cisplatin-induced renal injury by reducing ROS levels and inhibiting nuclear factor- $\kappa \mathrm{B}$ activation (30). The present study aimed to investigate the reno-protective effects of low- and high-dose GEN treatment in T1DM model rats. The results of the present study demonstrated that the renal index was decreased in rats receiving a low-dose of GEN, although there were no significant differences in $\mathrm{BW}$ and $\mathrm{KW}$; $\mathrm{KW}$ and renal index were decreased in rats treated with a high dose of GEN. BUN, 24-h urine protein, SCr, LPO and MDA were decreased, while T-AOC and SOD activities were increased in low-dose GEN-treated rats, and further improvements were observed in rats receiving a higher dose. In addition, western blotting results also demonstrated that GEN increased the protein expression of Nrf2, HO-1 and NQO1. These results suggested that GEN treatment may protect the diabetic kidney from oxidative stress by activating the Nrf2/HO-1/NQO1 signaling pathway. However, GEN treatment exhibited no effects on FBG levels in this model. Taken together, these results indicated that GEN may have reno-protective effects in diabetic rats by alleviating renal hypertrophic alterations, inhibiting lipid peroxidation and restoring balance to the anti-oxidant system, and this effect is not relevant to reducing the FBG level in STZ-induced diabetic rats.

Previous studies have revealed that GEN treatment inhibits tyrosine kinase and has an anti-proliferative effect on many types of cells (31-33). GEN was reported to inhibit aortic smooth muscle cell proliferation in vivo and to abolish nucleoside uptake by cardiac fibroblasts in vitro $(22,34)$. GEN also inhibits the expression of connective tissue growth factor and epithelial-to-mesenchymal transition, which indicated that GEN may have an antifibrogenic effect in parathyroid hormone-induced renal disease (35). However, there is no obvious evidence that GEN exerts its antifibrogenic activity in the diabetic kidney. Therefore, an attempt was made to determine the anti-fibrotic effects of GEN on renal fibrosis in subsequent experiments.

Renal fibrosis, characterized by the continuous accumulation and excessive deposition of ECM, is a major pathological feature of chronic kidney diseases (36). Excess ECM, which is caused by an imbalance between synthesis and degradation, serves a key role in the pathogenesis of renal fibrosis (37). In renal fibrosis, the major components of ECM are interstitial collagens, including collagen IV, and its increased biosynthesis or reduced degradation leads to glomerular basement membrane thickening and renal insufficiency (38). Masson's trichrome staining is a conventional method for the detection of collagen deposition. In addition, the Hyp residue is the main component of collagen IV formation. The results of the present study demonstrated that renal CVF, Hyp content and collagen IV protein expression were increased in diabetic model rat kidney tissues, which suggested that diabetes may induce renal fibrosis. In GEN-treated diabetic model rats CVF, Hyp content and collagen IV protein expression were decreased, which indicated that GEN may be able to attenuate the pathogenesis of renal fibrosis in diabetic rats.

In fibrotic diseases, TGF- $\beta 1$ is synthesized by all cell types in the kidney and is a crucial pro-fibrotic mediator; the expression of TGF- $\beta 1$ is significantly upregulated in damaged kidneys in both animal models and patients $(39,40)$. TGF- $\beta 1$ directly induces the activation of ECM genes and suppresses the degradation of ECM (41). Futhermore, TGF- $\beta 1$ may induce tubular endothelial-to-myofibroblast differentiation and directly promote the proliferation of mesangial cells $(42,43)$. Active TGF- $\beta 1$ exerts its biological and pathological effects through the Smad-dependent pathway in kidney disease (44). Smads are crucial intracellular nuclear effectors of TGF- $\beta$ family members. Of these, Smad3 is phosphorylated by TGF- $\beta 1$, which regulates transcription of downstream target gene (45). Smad3 is highly phosphorylated during renal fibrosis (46). A number of studies have demonstrated that TGF- $\beta 1 /$ Smad3 signaling is a central pathway leading to fibrotic diseases, including liver fibrosis (47), myocardial fibrosis (48) and pulmonary fibrosis (49). The results of the present study also demonstrated that the mRNA expression levels of TGF- $\beta 1$, as well as the protein expression levels of TGF- $\beta 1$, Smad3 and p-Smad3, and the p-Smad3/Smad3 ratio were increased in STZ-induced diabetic rats, which indicated that the TGF- $\beta 1 / \mathrm{Smad} 3$ pathway may be activated in renal fibrosis, and this result was consistent with previous studies (50-52). Following either low- or high-dose GEN treatment, the expression levels of TGF- $\beta 1, \operatorname{Smad} 3$ and $\mathrm{p}-\mathrm{Smad} 3$ were decreased. Therefore, the present study hypothesized that GEN treatment may downregulate the TGF- $\beta 1 / \mathrm{Smad} 3$ pathway to attenuate renal fibrosis in diabetic rats.

In summary, results from the present study demonstrated that GEN may attenuate renal fibrosis and exhibit reno-protective effects in T1DM model rats. The beneficial effects of GEN treatment on kidney injury may be attributed to its ability to suppress oxidative stress by activating the Nrf2/HO-1/NQO1 pathway and to downregulate the TGF- $\beta 1 / \mathrm{Smad} 3$ pathway to regulate collagen IV protein expression. 


\section{Acknowledgements}

Not applicable.

\section{Funding}

The present study was supported by The Natural Science Research Project of The Education Commission of Anhui Province (grant nos. KJ2017A216 and KJ2018A0994) and The Natural Science Research Project of Bengbu Medical College (grant nos. BYKY1621ZD and BYKF1706).

\section{Availability of data and materials}

The analyzed data sets generated during the study are available from the corresponding author on reasonable request.

\section{Authors' contributions}

QJ, RY and LW produced substantial contributions to the conception and design of the present study. QJ, XFL and SFM performed the experiments. QJ and RY analyzed the data and wrote the manuscript. All authors read and approved the manuscript.

\section{Ethics approval and consent to participate}

The present study was approved by the Animal Ethics Committee of Bengbu Medical College (Bengbu, China).

\section{Patient consent for publication}

Not applicable.

\section{Competing interests}

The authors declare they have no competing interests.

\section{References}

1. Thompson CS: Diabetic nephropathy: Treatment with phosphodiesterase type 5 inhibitors. World J Diabetes 4: 124-129, 2013.

2. Luyckx VA, Tonelli M and Stanifer JW: The global burden of kidney disease and the sustainable development goals. Bull World Health Organ 96: 414-422, 2018.

3. Shields J and Maxwell AP: Managing diabetic nephropathy. Clin Med 10: 500-504, 2010.

4. Tominaga T, Abe H, Ueda O, Goto C, Nakahara K, Murakami T, Matsubara T, Mima A, Nagai K, Araoka T, et al: Activation of bone morphogenetic protein 4 signaling leads to glomerulosclerosis that mimics diabetic nephropathy. J Biol Chem 286: 20109-20116, 2011.

5. Fernandes SM, Cordeiro PM, Watanabe M, Fonseca CD and Vattimo MF: The role of oxidative stress in streptozotocin-induced diabetic nephropathy in rats. Arch Endocrinol Metab 60: 443-449, 2016

6. Yu JB, Shi J, Gong LR, Dong SA, Xu Y, Zhang Y, Cao XS and Wu LL: Role of Nrf2/ARE pathway in protective effect of electroacupuncture against endotoxic shock-induced acute lung injury in rabbits. PLoS One 9: e104924, 2014.

7. Ansari MA: Sinapic acid modulates Nrf2/HO-1 signaling pathway in cisplatin-induced nephrotoxicity in rats. Biomed Pharmacother 93: 646-653, 2017.
8. Lv S, Zhou Q, Xia Y, You X, Zhao Z, Li Y and Zou H: The association between oxidative stress alleviation via sulforaphane-induced Nrf2-HO-1/NQO-1 signaling pathway activation and chronic renal allograft dysfunction improvement. Kidney Blood Press Res 43: 191-205, 2018.

9. Ilkun $\mathrm{O}$ and Boudina S: Cardiac dysfunction and oxidative stress in the metabolic syndrome: An update on antioxidant therapies. Curr Pharm Des 19: 4806-4817, 2013.

10. Rochette L, Zeller M, Cottin Y and Vergely C: Diabetes, oxidative stress and therapeutic strategies. Biochim Biophys Acta 1840: 2709-2729, 2014

11. Arora MK and Singh UK: Oxidative stress: Meeting multiple targets in pathogenesis of diabetic nephropathy. Curr Drug Targets 15: 531-538, 2014.

12. Liu L, Shi M, Wang Y, Zhang C, Su B, Xiao Y and Guo B: SnoN upregulation ameliorates renal fibrosis in diabetic nephropathy. PLoS One 12: e0174471, 2017.

13. Palanisamy N, Kannappan S and Anuradha CV: Genistein modulates NF- $\mathrm{KB}$-associated renal inflammation, fibrosis and podocyte abnormalities in fructose-fed rats. Eur J Pharmacol 667: 355-364, 2011.

14. Meng XM, Tang PM, Li J and Lan HY: TGF- $\beta /$ Smad signaling in renal fibrosis. Front Physiol 6: 82, 2015.

15. Guo TL, Germolec DR, Zheng JF, Kooistra L, Auttachoat W, Smith MJ, White KL and Elmore SA: Genistein protects female nonobese diabetic mice from developing type 1 diabetes when fed a soy- and alfalfa-free diet. Toxicol Pathol 43: 435-448, 2015.

16. Maulik SK, Prabhakar P, Dinda AK and Seth S: Genistein prevents isoproterenol-induced cardiac hypertrophy in rats. Can J Physiol Pharmacol 90: 1117-1125, 2012.

17. Zhou P, Wang C, Hu Z, Chen W, Qi W and Li A: Genistein induces apoptosis of colon cancer cells by reversal of epithelial-to-mesenchymal via a Notch1/NF- $\mathrm{B}$ /slug/E-cadherin pathway. BMC Cancer 17: 813, 2017.

18. Bhattarai G, Poudel SB, Kook SH and Lee JC: Anti-inflammatory, anti-osteoclastic and antioxidant activities of genistein protect against alveolar bone loss and periodontal tissue degradation in a mouse model of periodontitis. J Biomed Mater Res A 105: 2510-2521, 2017.

19. Rajput MS and Sarkar PD: Modulation of neuro-inflammatory condition, acetylcholinesterase and antioxidant levels by genistein attenuates diabetes associated cognitive decline in mice. Chem Biol Interact 268: 93-102, 2017.

20. Mirahmadi SM, Shahmohammadi A, Rousta AM, Azadi MR, Fahanik-Babaei J, Baluchnejadmojarad T and Roghani M: Soy isoflavone genistein attenuates lipopolysaccharide-induced cognitive impairments in the rat via exerting anti-oxidative and anti-inflammatory effects. Cytokine 104: 151-159, 2017.

21. Gupta SK, Dongare S, Mathur R, Mohanty IR, Srivastava S, Mathur S and Nag TC: Genistein ameliorates cardiac inflammation and oxidative stress in streptozotocin-induced diabetic cardiomyopathy in rats. Mol Cell Biochem 408: 63-72, 2015.

22. Yu JY, Lee JJ, Lim Y, Kim TJ, Jin YR, Sheen YY and Yun YP: Genistein inhibits rat aortic smooth muscle cell proliferation through the induction of p2 $7^{\text {kipl. }}$ J Pharmacol Sci 107: 90-98, 2008.

23. Yang R, Jia Q, Liu XF and Ma SF: Effect of genistein on myocardial fibrosis in diabetic rats and its mechanism. Mol Med Rep 17: 2929-2936, 2018

24. Li WF, Yang K, Zhu P, Zhao HQ, Song YH, Liu KC and Huang WF: Genistein ameliorates ischemia/reperfusion-induced renal injury in a SIRT1-dependent manner. Nutrients 9: 403, 2017.

25. Elmarakby AA and Sullivan JC: Relationship between oxidative stress and inflammatory cytokines in diabetic nephropathy. Cardiovasc Ther 30: 49-59, 2012

26. Lan HY: Transforming growth factor- $\beta /$ Smad signalling in diabetic nephropathy. Clin Exp Pharmacol Physiol 39: 731-738, 2012.

27. Al Hroob AM, Abukhalil MH, Alghonmeen RD and Mahmoud AM: Ginger alleviates hyperglycemia-induced oxidative stress, inflammation and apoptosis and protects rats against diabetic nephropathy. Biomed Pharmacother 106: 381-389, 2018.

28. Sifuentes-Franco S, Padilla-Tejeda DE, Carrillo-Ibarra S and Miranda-Díaz AG: Oxidative stress, apoptosis, and mitochondrial function in diabetic nephropathy. Int J Endocrinol 2018: 1875870, 2018.

29. Mahmoodnia L, Aghadavod E, Beigrezaei S and RafieianKopaei M: An update on diabetic kidney disease, oxidative stress and antioxidant agents. J Renal Inj Prev 6: 153-157, 2017. 
30. Sung MJ, Kim DH, Jung YJ, Kang KP, Lee AS, Lee S, Kim W, Davaatseren M, Hwang JT, Kim HJ, et al: Genistein protects the kidney from cisplatin-induced injury. Kidney Int 74: 1538-1547, 2008.

31. Sobhy MM, Mahmoud SS, El-Sayed SH, Rizk EM, Raafat A and Negm MSI: Impact of treatment with a protein tyrosine kinase inhibitor (Genistein) on acute and chronic experimenta Schistosoma mansoni infection. Exp Parasitol 185: 115-123, 2018

32. Shafiee G, Saidijam M, Tavilani H, Ghasemkhani N and Khodadadi I: Genistein induces apoptosis and inhibits proliferation of HT29 colon cancer cells. Int J Mol Cell Med 5: 178-191, 2016.

33. Qi W, Weber CR, Wasland K and Savkovic SD: Genistein inhibits proliferation of colon cancer cells by attenuating a negative effect of epidermal growth factor on tumor suppressor FOXO3 activity. BMC Cancer 11: 219, 2011

34. Pillai MS and Shivakumar K: Genistein abolishes nucleoside uptake by cardiac fibroblasts. Mol Cell Biochem 332: 121-125, 2009.

35. Guo Y, Zhang A, Ding Y, Wang Y and Yuan W: Genistein ameliorates parathyroid hormone-induced epithelial-to-mesenchymal transition and inhibits expression of connective tissue growth factor in human renal proximal tubular cells. Arch Med Sci 9: 724-730, 2013

36. Nogueira A, Pires MJ and Oliveira PA: Pathophysiological mechanisms of renal fibrosis: A review of animal models and therapeutic strategies. In Vivo 31: 1-22, 2017.

37. Wang Z, Han Z, Tao J, Wang J, Liu X, Zhou W, Xu Z, Zhao C, Tan R and Gu M: Role of endothelial-to-mesenchymal transition induced by TGF- $\beta 1$ in transplant kidney interstitial fibrosis. J Cell Mol Med 21: 2359-2369, 2017.

38. Diez-Marques L, Ortega-Velazquez R, Langa C, RodriguezBarbero A, Lopez-Novoa JM, Lamas S and Bernabeu C: Expression of endoglin in human mesangial cells: Modulation of extracellular matrix synthesis. Biochim Biophys Acta 1587: 36-44, 2002.

39. Sutariya B and Saraf M: Betanin, isolated from fruits of Opuntia elatior Mill attenuates renal fibrosis in diabetic rats through regulating oxidative stress and TGF- $\beta$ pathway. J Ethnopharmacol 198: 432-443, 2017.

40. Song JH, Cha SH, Lee HJ, Lee SW, Park GH and Kim MJ: Effect of low-dose dual blockade of renin-angiotensin system on urinary TGF- $\beta$ in type 2 diabetic patients with advanced kidney disease. Nephrol Dial Transplant 21: 683-689, 2006.

41. Samarakoon R, Overstreet JM, Higgins SP and Higgins PJ: $\mathrm{TGF}-\beta 1 \rightarrow \mathrm{SMAD} / \mathrm{p} 53 / \mathrm{USF} 2 \rightarrow \mathrm{PAI}-1$ transcriptional axis in ureteral obstruction-induced renal fibrosis. Cell Tissue Res 347: $117-128,2012$.
42. Wu CF, Chiang WC, Lai CF, Chang FC, Chen YT, Chou YH, Wu TH, Linn GR, Ling H, Wu KD, et al: Transforming growth factor $\beta-1$ stimulates profibrotic epithelial signaling to activate pericyte-myofibroblast transition in obstructive kidney fibrosis. Am J Pathol 182: 118-131, 2013.

43. López-Hernández FJ and López-Novoa JM: Role of TGF- $\beta$ in chronic kidney disease: An integration of tubular, glomerular and vascular effects. Cell Tissue Res 347: 141-154, 2012.

44. Lan HY: Diverse roles of TGF- $\beta /$ Smads in renal fibrosis and inflammation. Int J Biol Sci 7: 1056-1067, 2011.

45. Hata A and Chen YG: TGF- $\beta$ signaling from receptors to smads. Cold Spring Harb Perspect Biol 8: pii: a022061, 2016

46. Zhao K, He J, Zhang Y, Xu Z, Xiong H, Gong R, Li S, Chen S and He F: Activation of FXR protects against renal fibrosis via suppressing Smad3 expression. Sci Rep 6: 37234, 2016.

47. Fan K, Wu K, Lin L, Ge P, Dai J, He X, Hu K and Zhang L: Metformin mitigates carbon tetrachloride-induced TGF- $\beta 1 /$ Smad3 signaling and liver fibrosis in mice. Biomed Pharmacother 90: 421-426, 2017.

48. Li X, Han D, Tian Z, Gao B, Fan M, Li C, Wang Y, Ma S and Cao F: Activation of cannabinoid receptor type II by AM1241 ameliorates myocardial fibrosis via Nrf2-mediated inhibition of TGF- $\beta 1 /$ Smad3 pathway in myocardial infarction mice. Cell Physiol Biochem 39: 1521-1536, 2016.

49. Qu Y, Zhang L, Kang Z, Jiang W and Lv C: Ponatinib ameliorates pulmonary fibrosis by suppressing TGF- $\beta 1 / \mathrm{Smad} 3$ pathway. Pulm Pharmacol Ther 34: 1-7, 2015.

50. Wang W, Zhou PH, Hu W, Xu CG, Zhou XJ, Liang CZ and Zhang J: Cryptotanshinone hinders renal fibrosis and epithelial transdifferentiation in obstructive nephropathy by inhibiting TGF- $\beta 1 /$ Smad3/integrin $\beta 1$ signal. Oncotarget 9: 26625-26637, 2017.

51. Feng J, Xie L, Kong R, Zhang Y, Shi K, Lu W and Jiang H: RACK1 silencing attenuates renal fibrosis by inhibiting TGF- $\beta$ signaling. Int J Mol Med 40: 1965-1970, 2017.

52. Liu R, Das B, Xiao W, Li Z, Li H, Lee K and He JC: A novel inhibitor of homeodomain interacting protein kinase 2 mitigates kidney fibrosis through inhibition of the TGF- $\beta 1 / \mathrm{Smad} 3$ pathway. J Am Soc Nephrol 28: 2133-2143, 2017. 